\title{
Impact of Access to Medicines on Public Health. Problems Associated with Access, Use, and Abuse of Antibiotics
}

Antonio G. Pisabarro ${ }^{1}$ and Denisse P. Rivera de la Torre ${ }^{2}$

1 Institute for Multidisciplinary Research in Applied Biology, Public University of Navarre, Pamplona, Spain. E-mail: gpisabarro@unavarra.es

2 Hospital Infantil del Estado de Sonora, Hermosillo, México. E-mail: denisseriverat15@gmail.com

\section{Summary}

Access to medicines is one of the essential problems in Public Health of low- and middleincome countries (LMICs). The World Health Organization (WHO) defines access to medicines as the possibility of "having continuously accessible and affordable medicines in public or private health facilities that are within a kilometer of the place of residence." Access to medicines, as defined by the WHO, is not fully guaranteed in many LMICs and even in many regions of high-resource countries. The WHO identifies several factors as determinants of limitations in the access to medicines: rational selection, affordable prices, sustainable financing, and reliable health services. The action on these factors makes it possible to improve universal access to medicines with consequent improvement in Public Health. Adequate access to antibiotics and vaccination will avoid a large part of the deaths caused by infectious diseases in the LMICs. However, the emergence of resistance and the difficulties in vaccination campaigns due to socio-political or cultural problems make it challenging to fight many easily treatable infectious diseases.

The use and abuse of antibiotics are inevitably associated with the appearance of resistances that make them ineffective. Thus, whereas limited access to antibiotics raises mortality rates from infectious diseases, generalized open access to them ends up eliminating their clinical value. Moreover, the contraction of research in this field for many years has reduced the success in discovering new drugs. Additionally, local market regulations, inadequate selection, inaccessible prices, especially for those of second and third-generation, inefficient health systems, and difficulties of administration and control of prescription compliance, especially in the case of combined therapies, are additional obstacles to universal access to antibiotics. In order to simultaneously improve access to antibiotics and keep resistances under control, it is necessary to develop training and education activities at different social levels (from patients to various Health Care Providers) to complement the national or supranational strategic plans.

Keywords: access to medicines; access to antibuotics; impact of access to medicines on public health; problems associated with access; use; abuse of antibiotics resistance; aware; Africa 


\section{1.- Introduction}

The history of humanity has proceeded through changes marked by simultaneous benefits and costs. The domestication of farm animals and the colonization of new wild spaces put our ancestors in contact with a plethora of new microorganisms and new infectious diseases, sometimes transmissible.

Additionally, the invention of agriculture about 10,000 years ago led to the formation of ever-larger stable human settlements that favored the emergence of epidemics caused by transmissible pathogenic microorganisms that found an open field of expansion in urban agglomerations. Since then, infectious diseases have been a factor of the genetic selection that has shaped our evolution. Until the onset of the antibiotics age in the second half of the 20th century and the development of mass vaccination programs for certain diseases, communicable and noncommunicable infectious diseases have been the leading death causes in human populations. This situation has dramatically changed in the last 80 years: a large number of previously lethal diseases can be treated now with antibiotics, many of them prevented by vaccination, and in one case, smallpox, a pathogen has been eliminated, and the disease disappeared.

However, this overall picture is not alike around the world. Infectious diseases remain the direct or indirect cause of a large proportion of the deaths in low- and middle-income countries (LIMCs). In many cases, these diseases would be easily tractable if adequate access to commonly used antibiotics were available; but such access may be difficult or impossible in these regions. The use of antibiotics inevitably produces the emergence of resistance in the microorganisms they target. This appearance is accelerated when the antibiotic is misused. The global rise of antibiotic resistance makes them ineffective in preventing the progress of infectious diseases and will mark in the future the decline of the current era of antibiotics.

In LIMCs, this combination of factors is particularly dramatic as, on the one hand, limited access to antibiotics increases mortality from infectious diseases in some areas of the world, and, on the other, widespread access to antibiotics speeds up the emergence of resistance and limits their usefulness. For this reason, LMICs may find themselves losing the possibility of profiting the era of antibiotics before it fades as a consequence of the appearance of resistance.

\section{2.- Global and local causes of mortality}

In 2016, there were 56.8 million deaths worldwide [1], and more than half of them $(53.8 \%)$ were due to ten leading causes (Ischemic heart disease, Stroke, Chronic obstructive pulmonary disease, Lower respiratory infections, Alzheimer's disease, and other dementias, Lung, trachea and bronchial cancers, Diabetes mellitus, Traffic accidents, Diarrheal diseases, and Tuberculosis). That year, the top three mortality causes responsible for $32.1 \%$ of global deaths were not infectious, and the only three infectious diseases of the top ten caused the $9.9 \%$ of deaths (Lower respiratory tract infections, 5.2\%; Diarrheal diseases, 2.4\%; and Tuberculosis, 2.3\%). This picture was substantially different from that of the year 2000 when four of the top ten causes of mortality were infectious (HIV/AIDS was then an additional top cause of mortality) and accounted for $16.7 \%$ of all deaths. That is, in this lapse of 16 years, the lethal burden of infectious 
diseases decreased to $59.3 \%$ compared to that of 2000, and HIV/AIDS disappeared as one of the leading causes of mortality.

If we make a similar analysis focusing on the regions classified as low-income by the World Bank, the picture is different. In these countries, six of the top ten causes of mortality in 2000 were infectious and caused $45.3 \%$ of the total deaths (Lower respiratory tract infections, 10.4\%; HIV/AIDS, 10.0\%; Diarrheal diseases, 9.6\%; malaria, 7.0\%; Tuberculosis, 4.4\%; and Measles, 3.8\%). Even more, the top five causes of mortality in these countries were infectious diseases. The panorama improved in 2016 when only five of the ten leading causes of mortality were infectious, the total burden of these diseases fell to $30.8 \%$ (68\% of the value registered in 2000), and Measles disappeared from the top 10 after massive vaccination campaigns. Although these results were encouraging, the situation in low-income countries was still far from that seen in countries with high economic resources where only lower respiratory tract infections appeared among the top ten causes of mortality, and infectious diseases caused only $4.3 \%$ of fatalities.

According to the WHO, the panorama in Africa was the worst, in average, among the low-income regions as the first six causes of mortality in 2000 were infectious and accounted for $48.1 \%$ of total deaths (HIV/AIDS, 12.2\%; Lower respiratory tract infections, 10.6\%; Diarrheal diseases, 9.7\%; Malaria, 7.3\%; Measles, 4.3\%; and Tuberculosis, 4.0\%). Although this situation had improved substantially in 2016 when deaths from the leading infectious diseases accounted for $35.1 \%$ of the total ( $73 \%$ of those registered in 2000), the top three death causes in the region were still infectious (Infections of the lower respiratory tract, HIV/AIDS and Diarrheal diseases). Measles, which in 2000 was the fifth leading cause of mortality in Africa causing $4.3 \%$ of the deaths, did not appear in 2020 among the top 20 causes of mortality of the continent and it is a spectacular case of a reduction of the death burden caused by an infectious disease (form more than 414,000 deaths in 2000 to 46,000 in 2016). Compared to the death load in Africa, the mortality from infectious diseases represented $4.5 \%$ in the Americas and $2.7 \%$ in the European WHO region, where these percentages have stayed constant for the last 16 years.

In summary, in low-income regions in general and Africa in particular, infectious diseases continue to be, despite the evident progress made, the leading cause of mortality, and this is mainly due to the poor access to antibiotics in this region. Furthermore, these regions have additional problems in water sanitation and hygiene, weak disease prevention policies, insufficient number of sanitary facilities, and widespread circulation of counterfeit drugs (each year, more than 169,000 childhood deaths caused by pneumonia are a consequence of counterfeit drugs).

\section{3.- Factors influencing access to medicines}

The World Health Organization defines Access to Medicines as the possibility of having continuous and affordable access to them in public or private health centers that are located closer than one kilometer from the place of residence [2]. According to this criterion, nearly two billion people worldwide do not have access to necessary medicines, which constitutes one of the essential public health problems of LIMC. Access to medicines, as defined by the WHO, is not fully guaranteed in many LMICs and even in many regions of high-resource countries either [3]. 
Furthermore, the concept of access to medicines must include the availability of specific pharmaceuticals at not only a given time, but also the growing importance of the maintenance of drug stocks to cope with the increasing number of long-term treatments such as combined therapies for HIV, tuberculosis or malaria, and treatments for hepatitis B or diabetes.

In order to mitigate the effects of the lack or limited access to medicines in many countries, it is necessary to strengthen their health systems and to have guiding criteria that allow the responsible use of the limited resources available and ensure that medicines reach the people who need them. There are several factors influencing access to medicines: a rational selection of the most appropriate for each condition, affordable prices, sustainable financing, and the existence of reliable health services [4]. The action on these factors allows improving the universal access to medicines with the consequent improvement of the general public health. In the specific case of antibiotics, an additional factor to consider is the lack of discoveries of virtually new antibiotics in recent years. Instead, the advances have occurred mainly as pharmacological modifications of existing ones.

Access to antibiotics varies by country and even within regions, and their rational use is hampered not only by their physical availability or economic affordability but also by cultural barriers, behaviors of health providers, and patients. In many cases, there are national or local administrative difficulties that prevent or delay the entry of drugs into different markets, which, together with the mismanagement of the available resources, leads to selecting the antibiotics to be prescribed with low scientific criteria. The insufficient availability of generics, the limited training on how to use antibiotics, the attitude of local patients, and marketing pressures lead to prescriptions based on availability rather than effectiveness. Moreover, patient self-prescription in free or almost free access to antibiotics environments leads to inappropriate use and the rise of antimicrobial resistance. Finally, it is also necessary to consider the difficulties related to the administration and compliance control of antibiotic prescriptions, especially in the case of combined therapies, which results in additional treatment deficiencies and a consequent increase in resistance [5].

Even though more than $95 \%$ of medicines, including most of the essential antibiotics, are out of patent and could be manufactured as generics, in many cases, many of them are not available because of several concurrent factors [6]. In some cases, the health services have limited experience in the regulations for using medicines whose patent has expired. Moreover, in many low-income countries, there are great difficulties in the local production of generic drugs due to deficiencies in the scientific or technological infrastructures and in equipment maintenance that require specialized technical assistance from abroad. All this increases production costs and reduces the savings associated with the production of the generic.

Additionally, budgetary constraints in hospitals, health services, and local health systems and infrastructure make it difficult to deliver medicine to millions of people. Access to medicines also depends on procurement practices for supplies, tax and tariff policies, price increases that occur throughout the supply chain, and on the strength of national drug regulatory authorities. Likewise, difficulties for the commercialization of drugs are also frequently observed due to local regulations. Finally, in some cases, international 
conventions for the control of narcotic drugs can be a barrier to the circulation of medicines between countries.

Drugs in general and antibiotics, in particular, must be affordable for users, and their prices must be transparent. In the absence of adequate public funding, the use of drugs causes a high expense for patients, limiting their accessibility. The WHO estimates that close to $90 \%$ of patients in low-income countries buy the prescribed medications directly. The part of the family budget dedicated to medicines varies significantly depending on the level of economic development of the country, reaching $60 \%$ of health spending in some LIMC compared to $18 \%$ in OECD countries [7]. On the other hand, the increase in resistance to antibiotics necessitates the use of higher-priced products that are sometimes inaccessible (especially those of second and third-generation antibiotics), leading to an increase in the frequency of incomplete treatments and an increased risk of new resistance appearing.

In many cases, the lack of price transparency and its increase in the marketing chain means that the lower price of generic products does not reach the consumer, who, also, frequently shows mistrust about their real quality of generics aggravated by the presence of counterfeit drugs. Finally, the recent changes in the poverty map in many regions of the world have led to the estimation that $70 \%$ of the world poor now live in middleincome countries that are losing their eligibility to receive support from mechanisms such as the Global Fund to Fight AIDS, Tuberculosis, Malaria, and Gavi the Vaccine Alliance. These changes in eligibility necessitate changes in government policies to compensate for the resulting problems.

In addition to being affordable and of good quality, medicines must also be safe, requiring quality control through active pharmacovigilance systems and secure management of reliable supply chains to protect populations of substandard or falsified.

The specificity of antibiotics is due to their selective action on a molecule, system, or structure of the pathogen against which it is active. This site of action of the antibiotic is called a target. During the last decades, there are only very few new antibiotic targets, so the vast majority of advances in this field are oriented to other aspects of antibiotic pharmacology. Because of the increase in resistance that we will study later, the lack of new antibiotic targets is a limiting factor in the availability of antibiotics. Research on new antibiotics is mainly based on private capital and in a context in which the prices of antibiotics are so low that they impede profits, companies leave the market, which reduces investment in research and leads to further shrinkage of the market, and focuses research on prevalent diseases in countries with high economic resources. For this reason, investment in research on drugs for the treatment of diseases in disadvantaged areas and tropical or subtropical is meager.

\section{4.- The Model List of Essential Medicines and the AWaRe system}

WHO publishes every two years since 1977, the so-called Model List of Essential Medicines (MLEM) that includes a set of medicines selected by the WHO experts to help countries determine how to meet their priority health needs [8]. Since 2007, the WHO has also published the Model List of Essential Medicines for Children biannually to cover the needs of children under 12 years of age. 
The first MLEM included 220 medicines, and since then, the WHO expert committee on the use of essential medicines meets every two years to update it through a transparent process in which any entity (individuals, governments, pharmaceutical companies or associations) can propose the inclusion of a drug providing scientific evidence of its safety, efficacy, and cost-effectiveness. Furthermore, it is necessary to demonstrate that the proposed medicine is essential to meet priority health care needs and that it is available in sufficient quantities. Each proposal is reviewed by a Committee and open for discussion on the WHO website. After a time of exposure and discussion, the Committee meets in its entirety to compile the model list, before presenting it for final approval by the WHO Director-General.

WHO wants MLEM to help different countries develop their own national essential drug lists and to help national decision-makers reduce costs by making it easier for them to select essential drugs to meet their specific country's health needs. More than 150 countries have created their model lists of essential drugs following the outline of the WHO list. The MLEM provides updated information on the potential of the new products included in it and on the loss of effectiveness of other products that removed from the list.

The MLEM contains two sub-lists: the basic and the complementary. The basic list compiles the minimum drug needs for a primary health care system. It lists the most effective, safe, and cost-effective medicines for selected priority conditions using current data and estimates in future public health, and the potential for safe and cost-effective treatment. The supplemental list includes medications requiring specialized diagnostic or monitoring facilities, specialized medical care, or specialized training for their administration. Some drugs are on the supplemental list because of their higher costs or lower cost-effectiveness.

Since 2017, the WHO has included the classification of antibiotics in the Access, Surveillance, Reserve (AWaRe) categories in the MLEM to improve the use of antibiotics at local, national and global levels, to stop the appearance and spread of antimicrobial resistance and to ensure adequate treatment of infectious diseases [9].

The Access Group (AWaRe) includes antibiotics active against the 25 most frequent pathogens with the least tendency to generate resistance. Antibiotics in this group are first- or second-choices for empirical treatment of infectious diseases. These essential antibiotics must be available, affordable, and quality assured. The group includes 48 antibiotics, 20 of which are also in the MLEM. The WHO has established as a countrylevel goal that the Access Group's consumption of antibiotics represents at least $60 \%$ of total antibiotic consumption. This indicator permits to monitor access to essential drugs and progress towards Universal Health Coverage.

The Watching Group (AWaRe) includes antibiotics with a higher potential for generating resistance and includes most of the highest priority agents among antimicrobials of critical importance to human medicine. These drugs should be used only for a limited number of specific infectious syndromes and their use registered in administration programs and monitoring of the appearance of resistance. The surveillance group includes 110 antibiotics, 12 of which are also in the MLEM.

Finally, the Reserve Group (AWaRe) includes antibiotics and classes of antibiotics reserved for the treatment of confirmed or suspected infections due to multi-resistant 
organisms. Antibiotics in this group should be treated as a last resort option when antibiotics in other groups have failed. Antibiotics in the reserve group have a favorable risk-benefit profile and proven activity against high or critical priority pathogens according to the WHO priority list for research into new antibiotics. Reserve antibiotics must be accessible, but their use must be tailored to highly specific patients and settings when all alternatives have failed or are inappropriate. These drugs could be protected and prioritized as key objectives of national and international management programs involving monitoring and reporting of use, to preserve their efficacy. This group includes 22 antibiotics and, among them, seven of which are present in the MLEM. Finally, the AWaRe system includes 103 fixed-dose combinations of multiple broad-spectrum antibiotics whose use lacks enough evidence, nor is it recommended in high-quality international guidelines, so the WHO does not recommend their use in clinical practice.

The AWaRe classification can be a tool to track the consumption of antibiotics in each country by quantifying their relative or absolute use in each of the categories, which can help policymakers set performance targets and optimize the use of antibiotics in their countries. Comparison of absolute and relative consumption data by category at national and regional levels allows, for example, to detect excessive use of watch antibiotics and thus mark a target for antibiotic administration, to assess trends in use over time, and to measure the impact of the interventions carried out. Likewise, this tool can be useful for physicians to implement consumption monitoring activities at the local level and inform the development of antibiotic treatment guidelines.

\section{5.- The problem of the appearance of resistance to antibiotics}

The evolutionary mechanisms of all living things lead microorganisms to develop resistance to the antibiotics they find in their growth medium. As explained above, antibiotics owe their specificity to their molecular interaction with proteins or microbial structures, called antibiotic targets, that they inhibit or destroy. These targets are the result of the expression of their corresponding genes subjected to the mutation and selection processes that underlie the evolutionary process. Mutations in genes coding for targets that affect their interaction with the antibiotic without affecting the operation of the target itself, produce a resistance that renders the antibiotic ineffective. A resistant microorganism may be uncompetitive in an environment where the antibiotic is not present; however, it is competitive when it is, and in its presence, it will dominate the population of microorganisms. If the microorganism in question is responsible for a communicable disease, the use of the antibiotic will be ineffective for all patients affected by that pathogen. Since mutations occur randomly, and since populations of microorganisms are very high, the emergence of antibiotic resistance is an inevitable process over time. Interventions in this regard try to slow down the process of resistance emergence and maintaining stocks of antibiotics that can be used effectively for as long as possible.

There are four primary mechanisms by which a microorganism can acquire resistance to an antibiotic (see $[10,11]$ for a review). The modification of the target, already explained, is the first of them and occurs both in the case of acellular pathogens (viruses) and in cellular ones (bacteria and parasites). The other three mechanisms can be found only in cellular pathogens: first, the destruction or modification of the antibiotic by enzymatic 
systems of the pathogen (in the case of bacterial beta-lactamases that inactivate antibiotics from the group of penicillins and cephalosporins). Second, structural modifications that hinder the entry of the antibiotic into the pathogen (for example, the modifications in the porins of Gram-negative bacteria that prevent the entry of the antibiotic). And, third, and the development of pumping systems that expel the antibiotic out of the cell, lowering its effective concentration below the action threshold.

In the case of bacteria, in many cases, mutations that confer resistance to antibiotics are in genetic elements of bacteria (plasmids) that can be transferred horizontally between different individuals and even species (see [12] for a review). In this way, resistance to antibiotics can spread rapidly in the population and even moves to other microorganisms with which the resistant mutant coexists. Likewise, resistance can originate in a nonpathogenic bacterium and subsequently transfer resistance to a pathogen with which it comes into contact.

Although the emergence of drug resistance is unavoidable in the long term, the use of antibiotics can slow down or speed up the process $[13,14]$. The dose and duration of the treatments prescribed after the corresponding pharmacological studies reduce the frequency of the appearance of resistance significantly. On the contrary, the reduction of the doses or the shortening of the treatments favors the early appearance of resistant drugs because a low dose of an antibiotic selects the natural variants that have an individual tolerance to the compound and allow accumulation of progressive mutations that do not individually imply a high genetic load for the microorganism in question. The use of sublethal doses of an antibiotic is a way to obtain resistance to it in a laboratory.

Antibiotic resistance is increasing to dangerously high levels in all parts of the world and is one of the greatest threats to global health, food security, and development today [15]. The emergence of new resistance and its spread worldwide threaten our ability to treat common infectious diseases. Thus, a growing list of infections, such as pneumonia, tuberculosis, gonorrhea, and foodborne illness, is becoming increasingly difficult, and sometimes impossible, to treat as antibiotics become less effective. Furthermore, resistance is jeopardizing the achievements of modern medicine: organ transplants, chemotherapy, and surgeries, such as cesarean sections, become much more dangerous without effective antibiotics for the prevention and treatment of infections. Without urgent action, we are heading into a post-antibiotic era, in which common infections and minor injuries can kill again. The world urgently needs to change the way it prescribes and uses antibiotics [16].

As explained above, antibiotic resistance occurs when bacteria change in response to the use of these drugs, and it is necessary to explain to the population that it is bacteria, not humans or animals, that become resistant to antibiotics. These bacteria can infect humans and animals, and when the first-line antibiotics become ineffective to treat these infections, it becomes necessary to use more expensive medications, diseases last longer and can require more complicate, often long-term, treatments in hospitals, They increase health care costs, the economic burden on families and societies, and lead to higher mortality. Antibiotic resistance occurs naturally, but the misuse of antibiotics in humans and animals is accelerating the process. Selling antibiotics for human or animal use without a prescription worsens the emergence and spread of resistance. Similarly, in countries without standard treatment guidelines, antibiotics are often over-prescribed by doctors 
and veterinarians, and patients tend to require the prescription of antibiotics without any scientific basis for it.

Even if new drugs were developed, without behavioral changes, antibiotic resistance would remain a significant threat. Behavioral changes should also include actions to reduce the spread of infection through vaccination, hand washing, safer sex, and good food hygiene. Misuse and overuse of antibiotics accelerate the appearance od resistance as so does the inadequate prevention and control of infections. Given the ease and frequency with which people now travel, antibiotic resistance is a global problem, requiring efforts from all nations and many sectors.

\section{6.- Possible actions to prevent the appearance of resistance to antibiotics}

The emergence of resistance and obstacles in vaccination campaigns due to sociopolitical or cultural problems hinder the fight against many easily treatable infectious diseases. As a complement to national or supranational strategic plans, it is necessary to develop training and education activities at different social levels (from patients to different agents of the health administration) to achieve greater access to antibiotics and reduce the appearance of resistance and limit its spread [17].

In order to prevent and control the spread of antibiotic resistance, it is necessary to educate the population to use antibiotics only when prescribed by a certified healthcare professional and to avoid requiring antibiotics when indicated that they are not needed. The population must be aware of the importance of following the indications for the use of antibiotics and, especially, following complete treatments. Likewise, consumers should be informed never to share or use leftover antibiotics. The prevention of the appearance of resistance is closely associated with the prevention of infections. For this, it is necessary to develop campaigns aimed at promoting regular hand washing, the hygienic preparation of food, avoiding close contact with sick people, practicing relationships safer sex, and keeping vaccination programs up to date. In particular, proper food preparation is critical in areas where diarrheal diseases are one of the leading causes of mortality. There are five WHO critical points for preparing safe food: keeping food clean, separating raw and cooked, cooking thoroughly, keeping food at safe temperatures, using clean water and raw materials. Finally, it is necessary to inform the population of the convenience of choosing foods produced without the use of antibiotics to promote growth or prevent disease in healthy animals.

Politicians and social managers must orient their activity towards preventing the emergence of resistance by designing and implementing robust national action plans to deal with the emergence and spread of resistance to antibiotics, including the improvement of surveillance of resistant infections and the strengthening of infection prevention and control policies, programs, and measures. It is advisable to develop strategies to regulate and promote the proper use and disposal of quality medications. Likewise, it is the function of administrations to make information about the impact of antibiotic resistance available to citizens.

The action of health professionals in the fight against the appearance and spread of resistance can focus on preventing infections by ensuring that their hands, instruments, and working environment are adequately clean. Health professionals should prescribe and 
dispense antibiotics only when they are necessary (approximately half of the antibacterial antibiotic prescriptions are made to treat viral infections against which are ineffective) and following the usage guidelines described in the previous sections. Health professionals should diligently report antibiotic-resistant infections to the appropriate surveillance teams. Likewise, they need to explain to their patients how to take antibiotics correctly, the dangers of misuse of antibiotics, and the importance of taking infection prevention measures (vaccination, hand washing, safer sex, and covering the nose and mouth when sneezing).

The industry needs to move faster and more aggressively to research and develop new antibiotics, but new ways need to be put in place to stimulate research and development. While there are some new antibiotics in development, none are expected to be effective against the most dangerous forms of antibiotic-resistant bacteria [18]. In order to stimulate the discovery of new antibiotics, it is necessary to develop incentives for funding basic and clinical research, working with pharmaceutical companies, and stimulating research on new diagnoses that allow the broader and more responsible use of antibiotics. The WHO Global Action Plan on Antimicrobial Resistance (GAPAR) [19] requires the creation of new partnerships to encourage the development of antibiotics. The WHO and the Drugs for Neglected Diseases Initiative are working to create a global center for antibiotic research and development that will work closely with the pharmaceutical industry, universities, civil society, and global health authorities. The association will also ensure that the new drugs are affordable for all and will incorporate the need for conservation of new antibiotics in the development process.

The prevention of the appearance and spread of resistance is also the responsibility of the livestock sector [20, 21, 22]. The misuse of antibiotics in livestock, aquaculture, and crops is a crucial factor contributing to antibiotic resistance and its spread to the environment, the food chain, and humans. Producers need only administer antibiotics to animals under veterinary supervision. It is essential to avoid the use of antibiotics to stimulate growth or to prevent disease in healthy animals, the instruction of farmers on the convenience of vaccinating animals to reduce the need for antibiotics, and to use alternatives to antibiotics when available. The prevention of infections in animals involves improving biosecurity on farms and improving hygiene and animal welfare. Finally, this sector must promote and apply good practices in all steps of production and processing of food of animal and plant origin.

Combating antibiotic resistance is a priority for the WHO, who approved in 2015, the Global Action Plan on Antimicrobial Resistance (GAPAR) [19]. This plan aims to ensure, for as long as possible, the successful prevention and treatment of infectious diseases with safe and effective medicines used responsibly and accessible to all who need them. The GAPAR has five strategic objectives: to improve awareness and understanding of antimicrobial resistance, to increase awareness through surveillance and research, to reduce the incidence of infection, to optimize the use of medicines antimicrobials and ensure a sustainable investment that takes countries' needs into account by increasing investment in new drugs, diagnostic tools, vaccines, and other interventions.

\section{7.- The problem of counterfeit or low-quality antibiotics}


Right quality medicines are essential to promote public health. In some African countries, between 30 and $50 \%$ of the drugs in circulation are of poor quality. In many African regions, medicines are sold in open markets and by street vendors and come from contraband or illegal imports. Furthermore, most national manufacturers do not comply with good manufacturing, storage, and distribution practices for drugs [23].

Circulation of low-quality or counterfeit antibiotics is another threat to the power of drugs and another source of resistance [24]. These products are prevalent in countries where drug regulatory authorities are weak and in countries that bypass regulatory controls through Internet sales. The complex web network that characterizes the global production and distribution of pharmaceuticals, including a long and convoluted supply chain, puts all countries at risk. Products that enjoy lucrative commercial markets are particularly susceptible to counterfeiting, as are much-needed medicines and vaccines. Substandard and counterfeit drugs not only steal consumer incomes that pay for products that have little or no medical value. They cause harm by not solving a medical problem and have sometimes caused hundreds of deaths, especially when the products contain toxic ingredients. In July 2013, the WHO launched a Global System for Monitoring and Monitoring Counterfeit and Counterfeit Medicines in West Africa. Since then, the WHO has trained more than 400 regulators from 126 countries to use this system for rapid notification of substandard or counterfeit products uploaded to a secure WHO website. If the investigation confirms damage to health, the WHO responds within 24 hours, providing coordination and technical support in case of an emergency.

Human behavior is a determining factor in the development of drug resistance in certain border areas where there is the legal and illegal movement of people in populations with limited access to health care and who commonly self-medicate if they are sick. Substandard or false (counterfeit) drugs, the use of monotherapies of antibiotics to be prescribed in combination therapies to prevent resistance (case of artemisinin in Southeast Asia [25]), and lack of proper administration or full treatment are well-known problems that promote the emergence of resistant. Thus, for example, between 30 and $35 \%$ of antimalarials circulating in Africa and Southeast Asia are counterfeited, and about $10 \%$ of artemisinin combination therapies have expired at the time of use. A single dose of an artemisinin antimalarial will probably not select for transmissible resistance, but repeated administration of non-curative doses of drugs below the required standard levels necessary, fail in eliminating the parasite and promote the appearance of resistance [26].

\section{8.- Conclusion}

During the 20th century, medicine has made spectacular advances in the control of infectious diseases that had been the leading causes of mortality throughout history. This control has been due to the development of antibiotics since 1945 and to mass vaccination programs for certain diseases that have complemented other public health measures. However, the control of infectious diseases has not been homogeneous throughout the world: in countries with limited economic resources, infectious diseases continue to be the leading cause of mortality, mainly due to limited access to antibiotics in these regions. The socioeconomic conditions of low-income countries, on the other hand, make access to antibiotics especially onerous for patients, which leads to non-compliance with treatments and the acquisition of drugs in open markets that they dispense, on many 
occasions., low quality or counterfeit products. The misuse of antibiotics and the use of low-quality products favors the appearance of microbial resistance that nullifies the effectiveness of the drug. The emergence and spread of resistance are one of the leading long-term health problems worldwide, leading us to a post-antibiotic era. In low-income countries, the dramatic situation of entering the post-antibiotic era can occur without having been able to take advantage of the therapeutic potential of these drugs. The fight against the appearance and spread of resistance involves various groups in society. The contribution of all of them is essential to slow down the appearance and spread of resistance. Also, it is essential to remove as much as possible the administrative and structural barriers which difficult to spread the use of antibiotics (and other drugs) that are outside of patent protection and facilitate their local production. Guidance from WHO with its MLEM and with the AWaRe antibiotic classification system can help establish guidelines for these developments.

Despite all these difficulties, the evolution of the impact of infectious diseases in lowincome countries has been positive for the last fifteen years, with notable results in the case of measles and HIV / AIDS control. These two examples show the road to fight against other challenges in the control of infectious diseases in these regions.

There is an apparent public health problem with an uncertain and grayish picture: the treatments used for a growing number of infections have become less effective in many parts of the world as a result of resistance. This loss of effectivity places us in need of a standardized approach to measuring antimicrobial consumption to obtain a complete and comprehensive image of antimicrobial use and resistance and to be able to identify areas where corrective actions are needed. Reliable surveillance systems must provide reliable data that are easy to compare, exchange, or use locally, nationally, and globally are essential. Unfortunately, many low- and middle-income countries cannot establish and maintain systems to collect information on the consumption of these medicines.

It is imperative to gather efforts to build and improve antimicrobial surveillance systems, in consumption adapted to the national context, to optimize the use of antimicrobials to help ensure the sustainability of the national antimicrobial. An essential basis for countries to improve is to understand the patterns and quantity of antibiotics used, from which to generate policies, regulations, and interventions to optimize the use of such medicines, unifying joint efforts to fight antimicrobial resistance.

\section{9.- References}

1.- WHO. Disease burden and mortality estimates. Cause-specific mortality, 2000-2016. https://www.who.int/healthinfo/global_burden_disease/estimates/en/

2.- WHO. Access to affordable essential medicines. In: Millenium Development Goals. 2004. https://www.who.int/medicines/mdg/MDG08ChapterEMedsEn.pdf

3.- Chan M. 2017. Access to medicines: making market forces serve the poor. In Ten years in public health, 2007-2017. World Health Organization. Geneva: World Health Organization.

4.- WHO. Equitable access to essential medicines: a framework for collective action. 2004. https://apps.who.int/medicinedocs/pdf/s4962e/s4962e.pdf 
5.- Frost I, Craig J, Joshi J., Faure K, and Laxminarayan R. 2019. Access Barriers to antibiotics. Washington DC Center for Disease Dynamics, Economics \& Policy.

6.- Taylor DW. 2010 Pharmaceutical access in least developed countries: on-the-ground barriers and industry successes. The Cameron Institute.

7.- OECD. 2010. How much is too much? Value for money in health spending. In Value for Money in Health Spending. pp. 21-40.

8. - WHO. 2019. $21^{\text {st }}$ WHO Model lists of essential medicines. WHO Technical Report Series No 1021. www.who.int/medicines/publications/essentialmedicines

9.- https://adoptaware.org/

10.- Blair JMA, Webber MA, Baylay AJ, Ogbolu DO, and Piddock LJV. 2015. Molecular mechanisms of antibiotic resistance. Nat. Rev. Microbiol. 13: 42-51

11.- Munita JM, and Arias CA. 2016. Mechanisms of antibiotic resistance. Microbiol. Spectrum. 4: VMBF-0016-2015.

12.- Partridge SR, Kwong SM, Firth N, and Jensen so. 2018. Mobile genetic elements associated with antimicrobial resistance. Clinical Microbiol. Rev. 31: e00088-17

13.- Martínez JL, Baquero F, and Anderson DI. 2007. Predicting antibiotic resistance. Nat. Rev. Microbiol. 5: 958-965

14.- Furusawa C, Horinouchi T, and Maeda T. 2018. Toward prediction and control of antibiotic resistance evolution. Curr. Op. Biotechnology. 54: 45-49.

15.- WHO. 2014. Antimicrobial resistance: global report on surveillance. WHO Library Cataloguing-in-Publication Data

16.- Hernando-Amado S, Coque TM, Baquero F, and Martínez JL. 2019. Defining and combating antibiotic resistance from One Health and Global Health perspectives. Nat. Microbiol. 4: 1432-1442.

17.- WHO. 2018. www.who.int/news-room/fact-sheets/detail/antibiotic-resistance.

18.- López-Jácome E, Franco-Cendejas R, et al. 2019. The race between drug introduction and appearance of microbial resistance. Current balance and alternative approaches. Curr. Op. Pharmacol. 48: 48-56.

19.- WHO. 2015 Global action plan on antibiotic resistance. WHO Library Cataloguingin-Publication Data.

20.- Marshall BM, and Levy SB. 2011. Food animals and antimicrobials: impacts on human health. Clin. Mic. Rev. 24: 718-733.

21.- Tang KL, Caffrey NP, et al. 2017. Restricting the use of antibiotics in food-producing animals and its associations with antibiotic resistance in food-producing animals and human beings: a systematic review and meta-analysis. The Lancet Planet Health 1: e316-27

22.- WHO. 2017 WHO guidelines on use of medically important antimicrobials in foodproducing animals. Geneva: World Health Organization.

23.- Kelesidis T, Falagas ME. 2015. Substandard/Counterfeit Antimicrobial Drugs. Clinical Mic. Rev. doi:10.1128/CMR.00072-14

24.- WHO. 2017. WHO Global Surveillance and Monitoring System for substandard and falsified medical products. Geneva: World Health Organization. 
25.- Delepierre A, Gayot A, and Carpentier A. 2012. Update on counterfeit antibiotics worldwide; Public health risks. Médicine et maladies infectieuses 42: 247-255

26.- Dondorp AM, Newton PN, Mayxay M, et al. 2004. Fake antimalarials in Southeast Asia are a major impediment to malaria control: multinational cross-sectional survey on the prevalence of fake antimalarials. Tropical Med. Int. Health 9: 12411246.

27.- Imwong M, Jindakhad T, Kunasol C, et al. 2015. An outbreak of artemisinin resistant falciparum malaria in Eastern Thailand. Sci. Rep. 5: 17412 